\title{
A WIDEBAND, FIBER-OPTIC, TRUE TIME-STEERED ARRAY RECEIVER
}

\author{
Paul J. Matthews, Michael Y. Frankel, and Ronald D. Esman \\ Naval Research Laboratory \\ Code 5672, Optical Sciences Division \\ Washington, DC 20375-5338
}

\begin{abstract}
The first fiber-optically controlled true time-delay array receiver capable of wide instantaneous bandwidth is demonstrated. The one-dimensional array consists of eight spiral elements arranged in a sparsely populated, unequally-spaced array pattern designed to suppress grating lobes and give a narrow main beam. The receiver exhibited $\pm 60^{\circ}$ azimuth steering with no observable squint over a microwave component-limited bandwidth of 6-18 GHz.

\section{INTRODUCTION}

Phased array antenna systems have seen much recent development due primarily to the inherent advantages of an electronically steered beam over a mechanically steered beam. These advantages include speed, reliability, graceful degradation and longterm potential cost reduction due to the possibility of transmitting and receiving multiple independent beams from a common aperture. To date, many of these advantages have not been realized due to the limitations of traditional all-electronic control over the individual array elements. The major drawbacks have been size, weight, loss and narrow instantaneous bandwidths. Optical control techniques for phased array antennas are capable of mitigating many of the problems associated with all-electronic steering systems. In particular, optical techniques are able to provide the true timedelay (TTD) capability necessary for the ultrawide instantaneous bandwidths required for many current and future applications. A variety of optical TTD techniques have been proposed [1-7]. Many of these techniques require specialized or precisely matched
\end{abstract}

components which are not fully developed, have excessive losses, limited bandwidths and are not amenable to field-ready systems or applications. Additionally, many of the proposed techniques are suitable only for transmitter type applications. Only a handful of mostly narrowband receiver systems have been demonstrated $[3,8,9]$. We have recently demonstrated a two-dimensional wideband array transmitter using a dispersive fiber-optic prism architecture that alleviates many of the aforementioned problems [10]. This technique is readily adaptable to wideband array receivers.

Here, we demonstrate what we believe is the first true time-delay control of an ultrawideband array receiver. The system demonstrates $\pm 60^{\circ}$ azimuthal steering with no observable beam squint over a microwave component-limited bandwidth of 6 to 18 $\mathrm{GHz}$. As with the previously demonstrated transmit array beamformer, the technique is capable of being transitioned to real-world ultrawideband array receiver systems.

\section{SYSTEM CONFIGURATION}

The fiber-optic receiver system is shown schematically in Fig. 1. The microwave energy received by each of the eight array elements is amplified and fed to a series of electro-optic Mach-Zehnder modulators (MZMs) which intensity modulate a wavelength-tunable optical carrier. The optical carrier is provided by a tunable external cavity laser with a wavelength $(\lambda)$ range of 1470 to $1580 \mathrm{~nm}$. The optical source is amplified using an erbium-doped fiber amplifier before being corporately distributed to the eight MZMs. After modulation by the MZMs, the optical signals are fed to an eight-channel fiber-optic dispersive prism. 


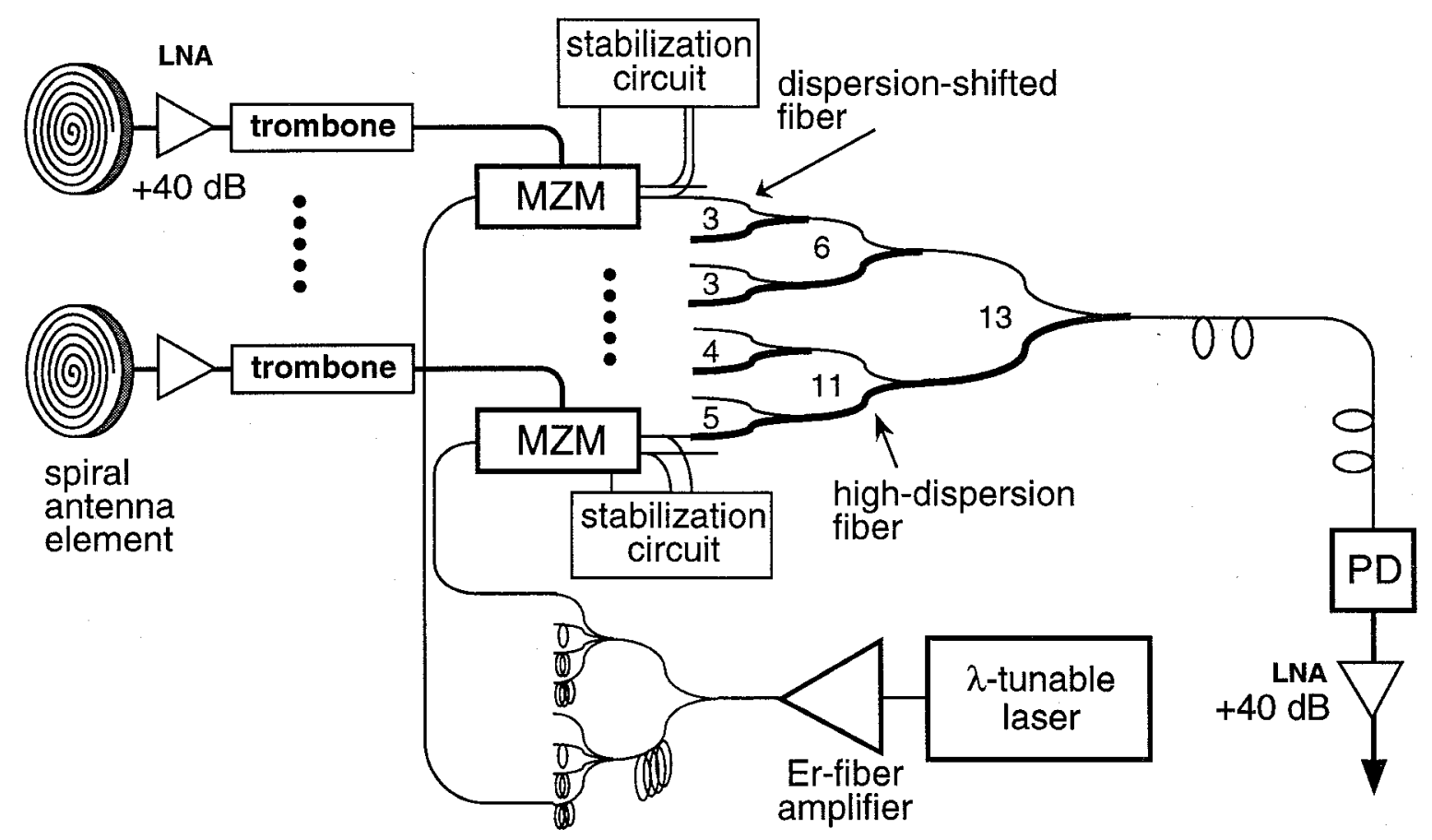

Figure 1. The fiber-optic true time-delay receiver schematic.

The prism provides a wavelength dependent time-delay for each element channel which is proportional to the position of the corresponding element in the array. The signals are then optically combined and fed to a high-speed photodiode (PD). Optical interference problems are minimized by differing the lengths of the corporate feeds to each MZM by more than the laser coherence length. Tuning the wavelength of the laser changes the group velocity of the signal propagating in each arm of the fiber-optic prism and alters the time-delay gradient across the array. Main beam steering is accomplished by tuning the wavelength to correspond to the conjugate, $-\theta$, of the received time-delay taper associated with energy arriving from an angle, $\theta$. The microwave signals from the eight elements add coherently at the PD forming a tunable matched filter.

The fiber-optic prism was fabricated in a binary "tree" type architecture using wavelength flattened 50/50 couplers. The nominal unit length of high-dispersion (HD) fiber was 55 meters, which corresponds to a $60^{\circ}$ angular steering with a wavelength detuning of $15 \mathrm{~nm}$ for the given receive array. The dispersion, $D$, of the HD fiber was approximately $-88 \mathrm{ps} / \mathrm{nm} \cdot \mathrm{km}$. The overall delays for each of the optical paths were equalized at a wavelength of $1550 \mathrm{~nm}$ using dispersion shifted (DS) fiber. A final timedelay trim was accomplished using microwave trombones before the MZMs. Fiber-optic attenuators were included in each array element path for amplitude matching.

The receiver was used in conjunction with eight matched spiral elements arranged in a sparsely populated, unequally-spaced pattern to suppress grating lobes. Such an arrangement also serves to narrow the main beam for improved angular discrimination. The elements were spaced 7.5, 7.5, 7.5, 10.0, $10.0,17.5$ and $12.5 \mathrm{~cm}$ apart and were capable of operating over the 2 to $18 \mathrm{GHz}$ band.

\section{RESULTS AND DISCUSSION}

The receive array was tested in a compact radar range. A network analyzer was used to drive a radiating horn antenna with a 2 to 18 
$\mathrm{GHz}$ bandwidth. The radiation from the horn was collimated using a microwave mirror and was detected by the fiber-optic receive system mounted on a rotation pedestal. The polarization mismatch between the radiating horn and the spiral elements resulted in a 3 $\mathrm{dB}$ power penalty. Receive patterns were taken over an angular span of $\pm 70^{\circ}$ in $0.25^{\circ}$ increments. The full 6 to $18 \mathrm{GHz}$ band was recorded in $1 \mathrm{GHz}$ increments.

The measured and calculated receive patterns for the array steered to broadside $(\lambda=1550 \mathrm{~nm})$ are shown in Figure 2 . The measured patterns were normalized by the measured single element pattern of the spiral element in order to show the receiver performance independent of the chosen radiating element. Good agreement is observed between the measured and ideal receive patterns over the full frequency range. Due to the sparsely-populated array pattern chosen, the sidelobe levels are at the 10 to $-15 \mathrm{~dB}$ level near the main lobe and increase to a $-5 \mathrm{~dB}$ level further away. Grating lobes begin to appear at about 13 GHz.

The array was then steered to $-60^{\circ}$ solely by de-tuning the laser wavelength to $\lambda$ $=1535 \mathrm{~nm}$. The results, shown in Figure 3, indicate wideband, squint-free operation over the complete 6 to $18 \mathrm{GHz}$ frequency range. Again, good agreement between the measured and calculated receive patterns is observed. Various other steer angles were also measured with similar results.

\section{CONCLUSIONS}

We have demonstrated the first wideband array receiver capable of time-delay steering over the 6 to $18 \mathrm{GHz}$ frequency band. The system is capable of squint-free steering over an angular span of $\pm 60^{\circ}$. Excellent agreement is observed between the measured and calculated receive patterns.

\section{ACKNOWLEDGMENTS}

This work was supported by the Office of Naval Research.
[1]. E. N. Toughlian and H. Zmuda, "A photonic variable RF delay line for phased array antennas," Journ. Lightwave Technol., vol. 8, no. 12, pp. 1824-1828, Dec. 1990.

[2]. I. Frigyes and A. J. Seeds, "Optically generated true-time delay in phased array antennas," IEEE Trans. Microwave Theory and Tech, vol. 43, pp. 23782386, Sept. 1995.

[3]. J. J. Lee, R. Y. Yoo, S. Livingston, V. I. Jones, J. B. Lewis, H. W. Yen, G. L. Tangonan and $\mathrm{M}$. Wechsberg, "Photonic wideband array antennas," IEEE Antennas and Propagation, vol. 43, pp. 966-982, Sept. 1995.

[4]. R. Soref, "Optical dispersion technique for time-delay beam steering," Appl. Opt., vol. 31, no. 35, 7395-7397, Dec. 1992.

[5]. D. Dolfi, F. Michel-Gabriel, S. Bahn and J. P. Huignard, "Two-dimensional optical architecture for time-delay beamforming in a phased-array antenna," Optics Lett., vol. 16, no. 4, pp. 255-257, Feb. 1991.

[6]. E. Ackerman, et al., "Integrated 6-bit photonic true time-delay unit for lightweight 3-6 GHz radar beamformer," in 1992 IEEE MTT-S Digest, pp. 681684, 1992.

[7]. N. A. Riza, "Transmit/receive time-delay beam-forming optical architecture for phased-array antennas," Appl. Optics, vol. 30, (32), pp. 4594-4595, Nov. 1991.

[8]. A.P. Goutzoulis, J.M. Zomp, B. K. Davies, I. Liberman, A. H. Johnson and P. D. Hricak, "Hardware-compressive, true time-steering for control of phased array antennas," Rome Laboratory Report, no. RL-TR-95-293, Jan. 1996.

[9]. A. S. Daryoush, R. Saedi, N. Samant, P. R. Herczfeld and M. Belaga, "Fiber optic fed C-band active phased array antennas," in 1992. IEEE MTT-S Digest, pp. 437-440, 1992.

[10]. M. Y. Frankel, P. J. Matthews and R. D. Esman, "Two-dimensional fiber-optic control of a true time-steered array transmitter," IEEE Trans. Microwave Theory and Tech., vol. 44, no. 12, pp. 2696-2702, Dec. 1996. 


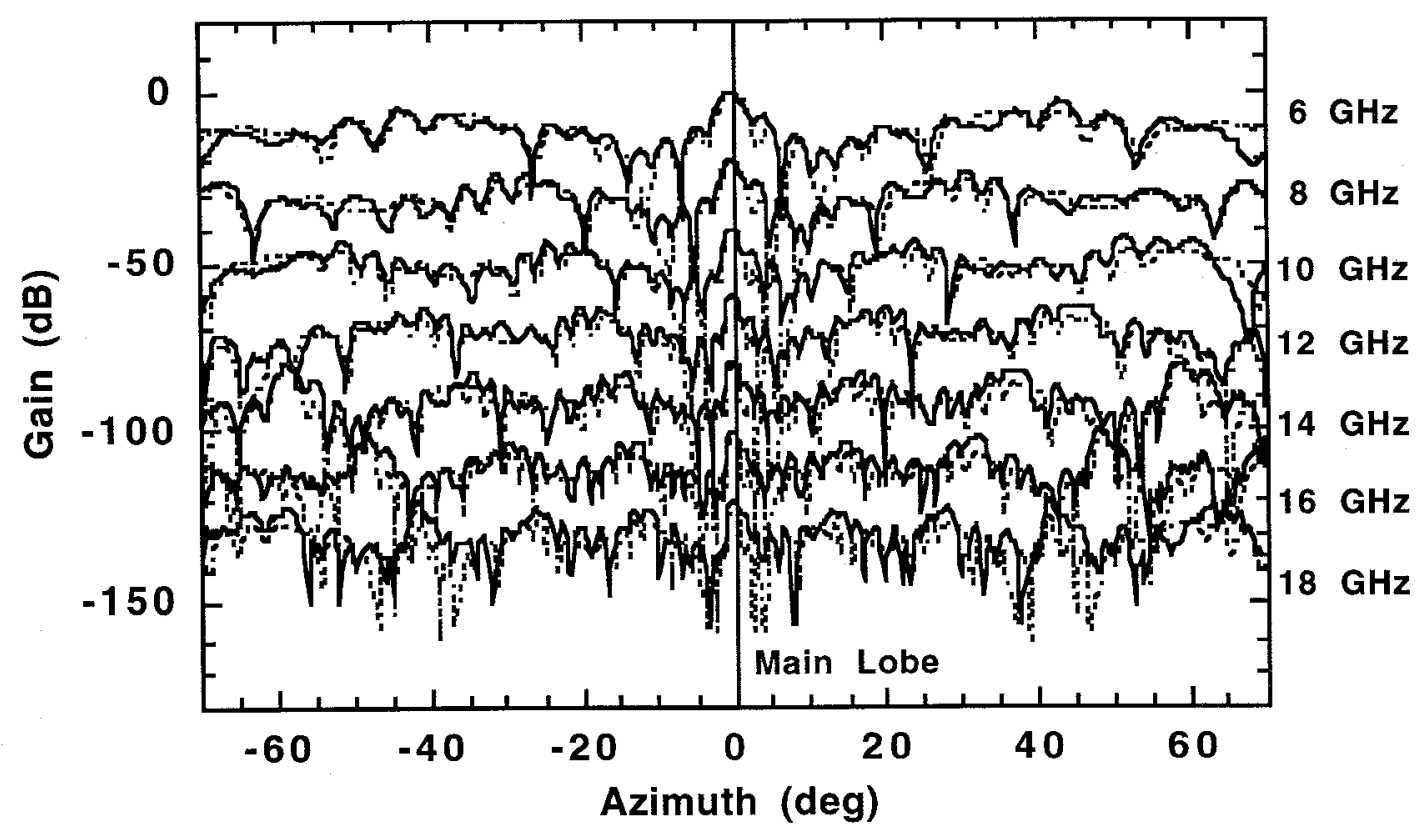

Figure 2. Comparison between the measured (solid) and calculated (dashed) antenna receive patterns with the laser tuned for broadside radiation. Frequencies of 6 , $8,10,12,14,16$ and $18 \mathrm{GHz}$ are shown with offsets for clarity. The array patterns have been normalized by the element pattern.

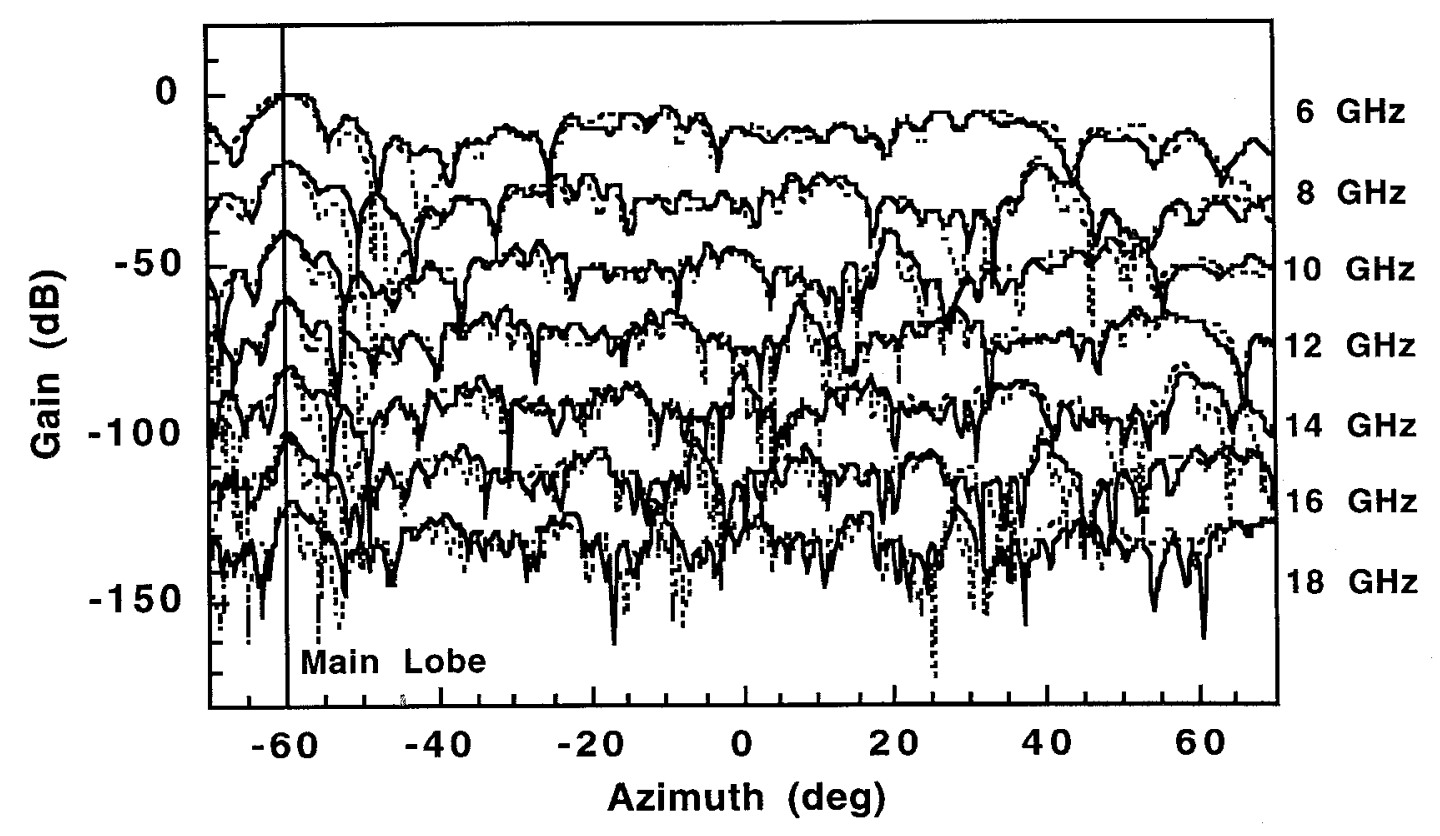

Figure 3. Comparison between the measured (solid) and calculated (dashed) antenna receive patterns with the laser de-tuned for $-60^{\circ}$ azimuth radiation. Frequencies of $6,8,10,12,14,16$ and $18 \mathrm{GHz}$ are shown with offsets for clarity. The array patterns have been normalized by the element pattern. 